\title{
The influence of reading speed and line length on the effectiveness of reading from screen
}

\author{
Mary C. Dyson and Mark Haselgrove \\ Department of Typography \& Graphic Communication, The University of Reading, \\ 2 Earley Gate, Whiteknights, Reading RG6 6AU, UK. \\ email: M.C.Dyson@reading.ac.uk
}

\section{(Received 16 August 1999 and accepted in revised form 5 January 2001)}

\begin{abstract}
With such a large volume of material accessible from the World Wide Web, there is an urgent need to increase our knowledge of factors influencing reading from screen. We investigate the effects of two reading speeds (normal and fast) and different line lengths on comprehension, reading rate and scrolling patterns. Scrolling patterns are defined as the way in which readers proceed through the text, pausing and scrolling. Comprehension and reading rate are also examined in relation to scrolling patterns to attempt to identify some characteristics of effective readers. We found a reduction in overall comprehension when reading fast, but the type of information recalled was not dependent on speed. A medium line length (55 characters per line) appears to support effective reading at normal and fast speeds. This produced the highest level of comprehension and was also read faster than short lines. Scrolling patterns associated with better comprehension (more time in pauses and more individual scrolling movements) contrast with scrolling patterns used by faster readers (less time in pauses between scrolling). Consequently, effective readers can only be defined in relation to the aims of the reading task, which may favour either speed or accuracy.
\end{abstract}

(C) 2001 Academic Press

KEYWORDS: comprehension; legibility; typography; reading rate; scrolling; skimming.

\section{Introduction}

A significant amount of reading is done at faster than normal speeds (Masson, 1982). This observation, based on the findings of a questionnaire, refers to the processing of printed texts. Subsequently, Muter and Maurutto (1991) extended the application to reading from screens, discussing the importance of investigating skimming from screens because of the widespread use of email, on-line abstracts, information retrieval, etc. Skimming has been defined simply in terms of how a text is read, i.e. sampling parts of a text whilst skipping other parts (Masson, 1985), and also related to the reading task, i.e. moving rapidly through text to locate specific information or gain the gist (Robeck \& Wallace, 1990). The rapid development of the World Wide Web (WWW) has contributed to the increased volume of material that we can read from screen. Web pages, in particular, are frequently skimmed, rather than read in detail (Horton, Taylor, Ignacio $\&$ Hoft, 1996). The outcome of this type of reading has received some attention, but 
Walczyk, Kelly, Meche and Brand (1999) claim that "few investigators have examined experimentally the effect of time limitations on reading comprehension".

A second area which requires investigation is the manner in which material is displayed on screen. An observation by Muter (1996) that we do not know how to optimize reading from screen is still true in relation to the layout of text. Although a significant volume of research has been conducted into the legibility of printed material (e.g. Spencer, 1968), a more limited number of studies has looked at reading from screen (reviewed by Dillon, 1992; Muter, 1996). As there are differences between reading from screen and print in terms of the process and outcomes of reading, it is important to extend the study of legibility on screen. There are few studies examining typographic variables on relatively recent display technology.

This context provides the basis for the current study which considers the nature of reading from screen. Can typographic factors or characteristics specifically associated with reading from screen (e.g. scrolling) be identified which affect reading rate and comprehension? Does increasing our speed of reading differentially affect the type of information we recall? What are the factors which influence how we scroll through text?

In an attempt to answer these questions, this study compares reading from screen at normal and fast speeds and focuses on a specific typographic variable, line length, to explore its effects on comprehension and reading rate. Comprehension is measured by a range of question types which assess the nature of material recalled. Patterns of scrolling movements are examined and we explore whether reading speed or line length affect these patterns. We also consider what aspects of scrolling patterns or reader characteristics might account for differences in reading rate and comprehension.

\section{Related research}

\subsection{READING SPEED, READING RATE AND COMPREHENSION}

The question of how comprehension is affected by changing the speed of reading was addressed by Poulton (1958). This study established that the amount remembered, a criterion of comprehension, increased significantly when reported speed of reading decreased from around 300 words/min to about 150 words/min. However, one of the conclusions of traditional studies on reading print conducted by Tinker in the 1930s and 1940s (summarized in Tinker, 1963) was that the fast reader tends to comprehend better.

Reasons for the apparent contradiction have been identified and explained by Carver (1990). Both negative and positive correlations between reading rate and comprehension have been reported as these are two different types of correlations: between-individual and within-individual. When an individual increases his or her reading rate, his or her comprehension decreases (e.g. Poulton, 1958), which is a within-individual negative correlation. However, people who naturally read fast also tend to demonstrate a high level of comprehension (e.g. Jackson \& McClelland, 1979), which is a between-individual positive correlation.

A starting point for research into reading from screen has been comparisons with print. The most common finding is that reading from screen is slower than reading print (e.g. Muter, Latremouille, Treurniet \& Beam, 1982; Gould \& Grischkowsky, 1984; Smith \& Savory, 1989). This suggests that reading from screen may be more difficult, although 
the specific factors responsible seem to have been rather difficult to identify (Gould, Alfaro, Barnes, Finn, Grischkowsky \& Minuto, 1987). An overall decrement in performance when reading from screen compared with print was found by Belmore (1985). Comprehension was worse and reading was slower in the screen condition. However, this result was attributed to participants' lack of familiarity with computers and reading from screen.

When speed of reading has been increased, this has surprisingly resulted in better comprehension when reading from screen than print (Muter \& Maurutto, 1991). However, this result could be accounted for by a speed-accuracy trade-off, as the rate of reading print was significantly faster than reading from screen. Dyson and Haselgrove (2000) also found a trade-off between reading rate and comprehension when readers were trained to read from screen at a faster speed.

In contrast, Walczyk et al. (1999) have found that mild time pressure, encouraging people to read slightly faster than normal from screen, can improve comprehension. The improvement is explained in terms of increased "mindfulness", i.e. mild time pressure increases motivation and effort. The relationship between reading rate and comprehension therefore appears to be dependent on the extent of reading acceleration. A small increase in reading rate may not impair comprehension but at some point increased reading speed appears to be at the expense of comprehension.

This discussion highlights the difference between natural variation in reading rate, both within and between individuals, and an experimental manipulation that influences reading speed. In some studies reading rate is a dependent variable, and in others an independent variable (cf. Breznitz, 1997). In this study, both are included by treating reading speed as an independent variable and reading rate as a dependent variable.

\subsection{NATURE OF COMPREHENSION}

Studies that have explored reading strategies when reading print have distinguished between the recall of details, more general ideas, and higher order processes requiring inferences (McConkie, Rayner \& Wilson, 1973; Wagner \& Sternberg, 1987). Johnson (1970) and other authors (e.g. Brown \& Smiley, 1977) have shown that the importance of units of text is related to their recall. Main ideas may be judged as important and easy to remember as they are interesting (Wade, Schraw, Buxton \& Hayes, 1993).

A detailed study of skimming stories in print, in which the nature of comprehension processes at different reading speeds was examined, was included in the Masson (1982) study. Although directed to skim for gist, participants were unsuccessful in retaining such information as reading rate increased. Important and unimportant information was equally likely to be skipped. Surface memory (i.e. recognition of specific wording of statements) was also more accurate at a normal reading rate, compared to skimming. A slightly different result was found by Just and Carpenter (1987), although in this study readers were not directed to read for particular information. Readers were asked to change from a rate of around 250 words per minute to around 600 or 700 words per minute. General comprehension or recall of the gist was not impaired, but recall of specific details was. McConkie et al. (1973) reached a similar conclusion that reading faster primarily reduces the amount of incidental information recalled.

The hypothesis that reading from screen at different reading speeds may differentially affect the type of information that is retained was tested by Dyson and Haselgrove (2000). 
Differences between question types were found at the normal reading speed with the best comprehension scores from higher order questions requiring inferences. Questions which required recall of the relative location of items within the text were particularly difficult, whatever the reading speed. However, there was no clear evidence from this study that rapid reading impairs the comprehension and recall of some types of information over other.

\subsection{LINE LENGTH}

Research into the relative legibility of different line lengths in print has led to recommendations that line lengths should not exceed about 70 characters per line (Spencer, 1968). Legibility has generally been measured by reading rate, with only a check on comprehension (Tinker, 1963), but analyses of eye movements can also make a contribution.

Rayner and Pollatsek (1989) have interpreted the rather confusing results from studies by Tinker and co-workers (summarized in Tinker, 1963) and come to a more specific conclusion on line length. They deduce that Tinker's work identified an optimal line length of 52 characters per line. The explanation given for the legibility of this moderate line length is that it is the outcome of a trade-off between two opposing factors. If line lengths are too long, the return sweeps to the beginning of the next line are difficult. If the lines are too short, readers cannot make use of much information in each fixation. Also, eye movement studies have revealed that readers decrease their saccade length, make more fixations, and increase fixation duration, when small windows are used. Therefore, reading short line lengths seems to be particularly inefficient.

Line length has also been found to affect reading rate on screen (Duchnicky \& Kolers, 1983; Dyson \& Kipping, 1998). Both studies, although using different display technologies, found that longer line lengths (about 75 and 100 characters per line, respectively), were read faster than very short lines. There may be less divergence between optimal line lengths in print and on screen if we consider the visual angles at normal reading distances. We tend to sit further away from the screen than from printed matter when reading (Gould et al., 1987); therefore, a longer line length on screen may subtend a similar visual angle to a moderate line length in print.

The effect of line length on reading rate may be dependent upon the overall reading speed, as speeding up reading may result in different patterns of eye movements. Masson (1985) has reviewed research on the characteristics of naturally fast readers and found that "superreaders" make fewer fixations. Although a distinction should be made between naturally fast readers and readers who are requested to increase their natural reading speed (see Section 2.1), normal readers' skimming has also been described in terms of sampling parts of a text which implies fewer fixations. As line length may also influence eye movements, an interaction between the two independent variables is possible.

The studies exploring line length (Duchnicky \& Kolers, 1983; Dyson \& Kipping, 1998) found no differences in comprehension but the same comprehension test was used in both studies. This test was primarily a check on comprehension and was not designed to detect differences in the recall of different types of information. Therefore, there remains the possibility that line length may affect certain types of comprehension which might be revealed if a more sensitive test is used. 
Based on the existing evidence described above, it is possible that line length may have a different effect on reading rate compared with comprehension. Treating reading rate and comprehension as separate dependent variables may demonstrate a speed-accuracy trade-off, but does not measure it. A combined measure of reading rate and comprehension has been used as an index of "effective reading performance" (Jackson \& McClelland, 1979). These authors multiplied reading rate by the comprehension score to produce their index. However, they do acknowledge that multiplication may not be the optimal formula, as sacrificing comprehension for very fast reading may exaggerate reading ability. Previous research has not explored the effect of line length on a combined measure of reading rate and comprehension.

\subsection{SCROLLING PATTERNS}

The mechanics of reading have been investigated by examining the way in which people move through a document (Harri-Augstein, Smith \& Thomas, 1982). Reading records were obtained through the use of a machine with text typed on a cylindrical roll of paper. Only two or three lines of text could be read at a time and readers controlled the rate at which they moved through the document by operating a handle. This movement was plotted on a graph. In principle, this method of presenting text is similar to current methods of scrolling through text on screen, although with a very small window. The analyses of "characteristic reads" (e.g. fairly rapid, more or less smooth, continuous read from beginning to end) made by Harri-Augstein et al. suggested avenues to explore in relation to scrolling patterns on screen.

Past research on the way readers move through a text on screen has mainly compared scrolling and paging (e.g. Hansen \& Haas, 1988; de Bruijn, de Mul \& van Oostendorp, 1992; Piolat, Roussey \& Thunin, 1997; Dyson \& Kipping, 1997, 1998). For example, Hansen and Haas (1988) and Piolat et al. (1997) identified some disadvantages of scrolling, compared with print or paged screen displays. Cues to the location of information (e.g. near the top of the page) are lost when text is scrolled within a window. However, scrolling is now commonly used when reading or skimming web pages. It is difficult to accommodate a paged format in browsers, as the amount of text per window or "page" is not fixed. Scrolling has been examined in relation to the ergonomics of different input devices (Zhai \& Smith, 1999) but the pattern of scrolling movements in relation to reading outcomes has received little attention.

An exception to this is the study by de Bruijn et al. (1992), which included an exploratory analysis of text manipulations. These were carried out to look at how people read text on screens of different sizes. Text manipulations were defined as near or far jumps, based on cursor or page up/down movements. They found that more text manipulations were used with a small screen and suggested that difficulties in integrating information in this condition resulted in reading and scrolling line by line. There is consequently a suggestion that the amount of text on screen, which can be manipulated by varying line length, may influence scrolling patterns.

The analysis by de Bruijn et al. (1992) formed the basis for studies by Dyson and co-workers, which have begun to examine scrolling patterns in relation to line length. Documents with long line lengths require less scrolling as there are fewer lines. Readers can therefore spend less time in scrolling movements which is likely to contribute to the 
faster reading rates at longer line lengths reported in Section 2.3. However, the time spent in scrolling movements is not necessarily added to a constant time (i.e. the pauses between scrolling movements). Data from Dyson and Kipping (1998) suggest that participants read whilst scrolling at shorter line lengths and not with longer line lengths. Readers therefore appear able to adjust their scrolling patterns according to the line length they are reading.

Due to the limited number of studies incorporating scrolling patterns, their relationships with other independent variables (reading speed) and dependent variables (reading rate and comprehension) have not yet been pursued. Given the flexibility which readers appear to have in adjusting their methods of scrolling according to circumstances, there may be changes in scrolling patterns with faster reading speeds. Making long scrolling movements appears to be a more efficient way of scrolling as Dyson and Kipping (1998) found that readers who made fewer discrete movements spent less time in scrolling. Furthermore, the scrolling pattern adopted for particular line lengths may also be influenced by reading speed.

With regard to reading rate and comprehension, questions remain as to whether scrolling patterns (or other factors associated with readers) may account for differences in these measures. Less frequent reading from screen may result in slower reading, although this suggestion is based on a study conducted when participants were generally fairly unfamiliar with computers (Belmore, 1985). It is likely that some scrolling patterns are more efficient and may therefore increase reading rate, but there is as yet no experimental evidence for this. If scrolling patterns are influenced by both reading speed and line length, and different scrolling patterns result in different reading rates, there may be an effect of line length on reading rate which depends on overall reading speed.

There is a slightly stronger case for predicting that scrolling patterns may affect comprehension. The way in which text is manipulated has been associated with a drain on the cognitive resources required for comprehension (Waller, 1986). Specifically in relation to scrolling, distraction may be caused by the mechanics of the movements (Hansen \& Haas, 1988; O'Hara \& Sellen, 1997). This proposal is consistent with Dyson and Haselgrove (2000) who found that the overall time spent pausing between scrolling was the best predictor of comprehension. When reading at normal speed, the readers with higher comprehension scores were those who spent more time pausing between scrolling and made fast and frequent scrolling movements. This pattern is likely to minimize the distraction from scrolling. A finding from Poulton (1958) may also be relevant when considering the relationship between pausing and scrolling. The context is rather different as Poulton controlled the presentation of text to his readers and new letters appeared on the right and disappeared on the left. However, he found that comprehension was better if there were breaks between reading where statements could be memorized, as compared with steady reading.

\subsection{RESEARCH QUESTIONS}

The results of experimental work on reading print and reading from screen provide a basis for the research questions in this study, but this work does not always provide sufficient grounds for firm predictions. Some of the questions are therefore exploratory in 
nature as new variables are investigated. In these circumstances, a number of possible outcomes are discussed. The questions are grouped according to the three dependent variables used in this study: comprehension, reading rate and scrolling patterns. A manipulation of reading speed (independent variable) compares participants reading faster than their normal reading speed with other participants reading at their normal speed. Individual reading rates (dependent variable) are measured within these two speeds.

\subsubsection{Comprehension}

(1) Does reading speed affect comprehension?

We expect less material will be recalled at the fast speed as this manipulation requires readers to substantially increase their normal reading speeds.

(2) Does an individual's reading rate affect their comprehension?

In contrast to the above prediction, we expect those individual readers who read faster (i.e. have a faster reading rate) to understand more.

(3) Does line length affect comprehension?

The effect of line length on comprehension is less certain as previous research has not been able to detect differences in the amount of material recalled from documents of different line lengths. The only differences have been dependent on the individual's reading rate. Using a more sensitive measure of comprehension may detect variation, but the direction of these differences is difficult to predict.

One outcome is that readers may vary their reading rate to maintain a relatively constant level of comprehension across line lengths (i.e. speed-accuracy trade-off). An alternative outcome is that a moderate line length may improve comprehension. This is based on the account of problems in reading both short and long lines arising from eye movement analyses. Such difficulties may detract readers from the comprehension process. Another way in which readers may be detracted is through the mechanics of scrolling. A further possible outcome is therefore the longer the line, the better the comprehension as less time need be spent scrolling.

(4) Is some material recalled better than other?

We expect readers to have more difficulty in recalling detail than more general facts. Questions which require recall of the location of material are predicted to be answered the least well. Higher order questions requiring inferences are expected to be among the easiest to answer.

(5) Does reading speed affect the type of material recalled?

Studies addressing this question have resulted in different outcomes. However, based on the differences that have been identified, detailed information might be relatively more disadvantaged when reading fast.

\subsubsection{Reading rate.}

(6) Does line length affect reading rate?

Previous experimental work leads to the prediction that longer lines will be read faster, which may be partly attributable to spending less time in scrolling movements. However, findings from the legibility of print would predict faster reading at medium line lengths. 
(7) Does the effect of line length on reading rate depend on the overall reading speed? The influence that overall reading speed might have on the effect of line length on reading rate is uncertain. However, it is possible that skim reading is facilitated by some line lengths and hindered by others, and this pattern may not be the same when reading at a normal speed. When required to read at the fast speed, it may be easier to speed up when there are fewer lines to scroll through at long line lengths. At normal reading speeds, this difference between line lengths may be less pronounced.

(8) Does line length affect the combined measure of reading rate and comprehension?

This question explores the possibility of a speed-accuracy trade-off by combining the two measures, as recommended by Jackson and McClelland (1979). There is no direct evidence from previous studies to provide the basis for predictions and these naturally depend upon the outcome of the individual variables (i.e. research questions 3 and 6). One possibility is a speed-accuracy trade-off across line lengths resulting in no differences in the combined measure [one of the outcomes proposed for research question (3)]. Alternatively, if eye movements are disrupted by some line lengths, this could affect both measures and result in differences between line lengths in the combined measure. This outcome could also be the result of scrolling patterns affecting both measures in a similar way.

\subsubsection{Scrolling patterns}

(9) Does reading speed affect scrolling patterns?

As fast reading imposes time pressure, readers may need to modify their methods of scrolling through documents. An obvious way to do this is to spend less time in pauses between scrolling movements. Time spent in scrolling can also be reduced by limiting the number of individual scrolling movements. If readers are aware of this outcome then one strategy to help increase speed of reading would be to make fewer, longer scrolling movements. However, time pressure may work against this strategy as more frequent, shorter scrolling movements may give the impression of moving through the document more rapidly and thereby speeding up the reading process.

(10) Does line length affect scrolling patterns?

Line length is likely to modify the process of scrolling to cater for the different amounts of text visible on screen at different line lengths and/or to adjust for the number of lines which must be scrolled through at different line lengths. At shorter line lengths, less time may be spent in pauses between scrolling movements, to compensate for longer scrolling time. Scrolling movements may also be longer with short lines to bring up an equivalent amount of new text as a shorter movement with longer lines.

(11) Does the effect of line length on scrolling patterns depend on reading speed?

One outcome is that fast reading has a consistent effect across line lengths, reducing the time spent in pauses [research question (9)], i.e. no interaction. Alternatively, if both time pressure and short line lengths serve to reduce the length of pauses, this may lead to a floor effect whereby pauses cannot be further shortened. This would result in a greater difference between the time spent in pauses at normal and fast speeds for longer line lengths than short lengths.

A similar pattern might also be found with scrolling movements. Reading speed may have a consistent effect across line lengths, i.e. no interaction. However, an alternative outcome is that a maximum length of scrolling movement is reached when reading short 
lines fast, resulting in a smaller difference between reading speeds in the length of scrolling movements at $25 \mathrm{cpl}$ compared with longer line lengths.

(12) What might account for differences in reading rates between readers?

Measures based on scrolling patterns are combined with personal characteristics to assess their individual contributions. A possible outcome is that people who report that they are less frequent readers of text on screen are slower readers. In relation to scrolling patterns, obvious means of reducing reading times are to decrease the time spent in pauses and/or scrolling time.

(13) What might account for differences in overall comprehension between readers?

Based on existing findings, the most likely factor to affect comprehension is the amount of time spent in pauses. We would therefore expect readers who spend longer in pauses to have better comprehension.

\section{Method}

\subsection{PILOT WORK}

Two pilot studies were carried out to establish an objective basis for devising comprehension questions which differed in terms of the nature of material requiring recall. These are described in more detail in Dyson and Haselgrove (2000). The first pilot identified the most important "units" of documents, which provided a criterion for developing questions that addressed the main issues, as opposed to details or incidental facts. The second pilot tested the accuracy of these questions and made modifications where appropriate.

\subsection{EXPERIMENTAL DESIGN}

The independent variables include one between and one within subject variable. Reading speed with two levels, fast and normal, is a between subject variable and line length with three levels measured in characters per line (cpl) is within subject.

The dependent variables are comprehension, reading rate and scrolling patterns. Comprehension is measured by a set of multiple choice questions covering a range of types of material and the questions are described in detail in Section 3.4. Reading rate is computed as the number of words per second. Scrolling patterns are measured by the time spent in pausing, time in scrolling, the length of the first pause and the number of scrolling movements.

An initial practice trial was introduced at the beginning of the experiment, using a document with a line length of $60 \mathrm{cpl}$. This practice familiarized participants with the type of questions they would be asked and ensured they knew how to move through documents and call up the next document. Each participant in either the fast or normal reading speed group then read six documents. These were presented in three blocks of two documents, each pair at the same line length. Two trials per line length were used as this increases the reliability of the results whilst keeping the total length of the experiment to a reasonable time. This design is summarized in Table 1. The pairing of line length with documents and their order of presentation were counterbalanced as far as possible, based on a Greco-Latin square design. 
TABLE 1

Between and within subject variables

\begin{tabular}{|c|c|c|c|c|c|c|c|c|c|c|c|c|c|c|c|}
\hline Between subject & Reading speed & \multicolumn{7}{|c|}{ Fast } & \multicolumn{7}{|c|}{ Normal } \\
\hline Within subject & Line length (cpl) & 60 & 2. & & 5 & & & & 60 & & & 5 & & 1 & \\
\hline & Documents & $\mathrm{P}$ & 1 & 2 & 3 & 4 & 5 & 6 & $\mathrm{P}$ & 1 & 2 & 3 & 4 & 5 & 6 \\
\hline
\end{tabular}

$\mathrm{P}=$ practice document.

\subsection{PARTICIPANTS}

Thirty-six volunteers took part in the study. They were recruited locally through advertisements within the University of Reading. Most participants were undergraduates or postgraduate students at the University and were given a small remuneration for their time.

Participants were randomly assigned to one of the two reading speeds. In the fast speed group, $67 \%$ of participants were between 18 and 24 years old; in the normal speed group, $78 \%$ were in this age band. The remainder in both groups were between 25 and 44. All reported using computers in some way: those using them for both leisure and work comprised $39 \%$ of the fast speed group and $78 \%$ of the normal speed group; those using computers just for work comprised $61 \%$ of the fast speed group and $17 \%$ of the normal speed group; one person in the normal speed group reported using computers only for leisure. On average, the group reading at the fast speed estimated that they read less frequently, both print and screen, than the normal reading speed group, but these differences were small. There was also a slightly larger range of responses from the participants allocated to the fast reading speed group. We would not expect differences of this size to affect comparisons between measures of performance at the two speeds.

\subsection{MATERIALS}

A number of articles that were considered to be of general interest were selected from the magazine National Geographic. (Permission was obtained to use these articles.) The documents were edited to be approximately equal length (up to 1000 words) by deleting text from the end, ensuring that the story line remained intact.

Six types of questions were identified. Title questions (T) asked which of the alternative titles best fit the text. Main idea questions (MI) covered one of the main themes in the text. These two types aimed to require the reader to make inferences about what was read, described by McConkie et al. (1973) as "higher order" questions. Structure questions (S) asked about the order of items within the text, i.e. what came before or after a particular item. Main factual questions (MF) asked about relatively important aspects of the text. Both structure and main factual questions therefore required the recall of general ideas. Incidental questions (I) concerned details in the text and recognition questions (R) asked whether the extract had appeared in the text. Both were looking for the recall of specific details. 
The first five question types were written as multiple-choice questions, with three alternative answers. Only one title, main idea and structure question could be written for each document, as these tended to encompass the whole document. However, it was possible to develop three main factual and three incidental questions for each text. In each case, one of the incidental questions referred to a number in the document. The recognition questions consisted of 10 short extracts, of which five were taken from the text that had been read, and five from the same source material, but from a part that had not been read. This ensured that the theme and writing style of the extracts were similar. An example of each type of question is given in the appendix.

\subsection{PROCEDURE}

The monitor was placed on a stand and adjusted to a fixed height that was intended to suit most people. Participants were able to choose where they sat in relation to the monitor, whilst being able to reach the keyboard. The mouse was placed out of reach as participants interacted solely via the keyboard, which was covered so as to reveal only those keys that participants needed to use. These keys displayed the documents, scrolled through the text and removed the text from the screen, when reading was complete. Cursor keys were chosen as the means of moving through the document to provide a simple means of interaction. We hoped that this would not require any practice to coordinate the hand and screen action, and participants would be able to position text accurately, i.e. not under or overshoot. Scrolling with a mouse, or similar device, requires precise control of positioning and speed of movement to get text in the right place (Zhai \& Smith, 1999). Although the WIMP interface is extremely common, we wished to be able to accommodate inexperienced computer users. As it turned out, none of the participants were in this category. This method of interaction also enabled a precise record of patterns of movement through the document.

The text was black and displayed in a window with a white background that was positioned on the left of the screen. The remainder of the display, to the right of the window, was light grey. The width of the window varied to accommodate different line lengths, with a margin of about 0.5 in on the right and left of the text. Items such as tool bars, menu bars and scroll bars were removed from view. When participants pressed the down arrow key, the cursor moved down line by line and the up arrow key moved back up.

The text was displayed in Arial in 10 point with 12-point interlinear spacing. There was an additional 12 points between paragraphs. This method of indicating new paragraphs is typically found on web pages. The text was left aligned, with no justification or hyphenation. Figure 1 illustrates the layout of the screen with a document of 55 characters per line $(55 \mathrm{cpl})$. At $25 \mathrm{cpl}$, the documents extended over about 4.5 screens; at $55 \mathrm{cpl}, 3$ screens and at $100 \mathrm{cpl}$, about 1.7 screens.

In order that participants in the fast reading speed condition could achieve a faster reading speed, which was relative to their normal speed, a period of training was introduced at the beginning of the experiment. In this training stage, participants were asked to read an initial document at their normal, comfortable reading speed. They were then asked to speed up their reading and try to read the next document at twice this speed, i.e. taking half as much time. Having read the second document, a message box 


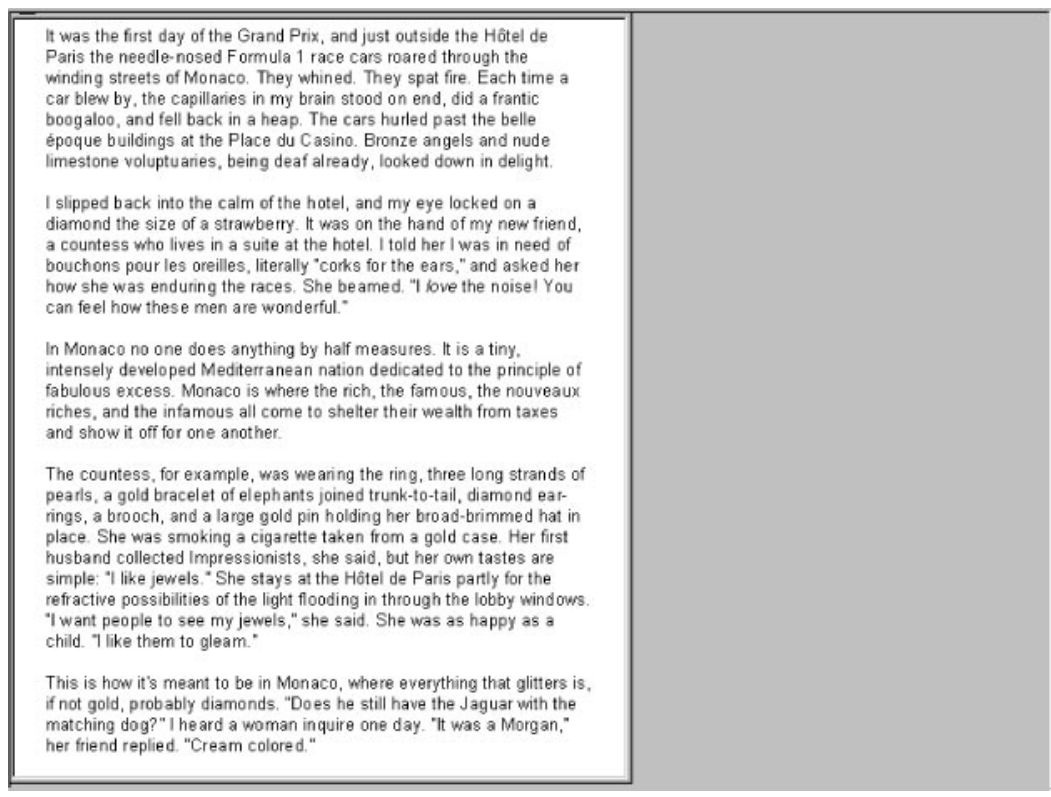

Figure 1. Example of screen layout with document of $55 \mathrm{cpl}$ (50\% actual size).

indicated whether or not they had read twice as fast. If they had, they proceeded to the testing stage of the experiment. If they had not read fast enough, they were given some indication of how much faster they needed to go. They were then asked to read another document. On each trial, a new document of approximately 500 words was used. This prevented participants from using the familiarity of the text to read faster. No comprehension questions were included in this stage.

The procedure continued until the participant either reached the target speed or had read eight documents. In the latter case, if the participant's reading time was no more than $70 \%$ of their first reading time, they continued with the experiment. If they had not been able to speed up their reading sufficiently, they did not take any further part in the experiment. (This happened very rarely.)

A more liberal criterion of $70 \%$ rather than $50 \%$ was introduced at this stage in the experiment as pilot work had indicated that a significant number of people found it difficult to reach a rate twice as fast as normal reading speed. If we had retained the criterion of twice normal reading speed, we would have selected a sample that might not be representative of typical readers from this population. Our aim was not to identify potential speed readers, but to attempt to simulate rapid or skim reading which would be within peoples' natural capabilities. The target of 50\% reading time set at the beginning of the training was retained to encourage faster reading, which would provide a clearer distinction between normal and fast reading.

Instructions to participants were to silently read a series of documents displayed on screen. No indication was given as to how the document should be read, as we hoped to discover which types of information are generally recalled at different reading speeds and 
line lengths. When participants were ready to start reading a document they pressed a key which displayed the document on screen and recorded the time they started. As they read and moved through the document with the cursor up and down keys, the time of each keystroke (and whether it was up or down) was recorded by the programs running the experiments. Participants appeared to have no difficulty in using the cursor keys to scroll, even though this may not have been their usual means of scrolling. Each participant's record of keystrokes provided the raw data from which scrolling pattern descriptions were derived. When they had read through to the end of a document, they pressed a second key which resulted in a blank screen and the time was recorded. Participants were then given a set of written questions on paper, which they needed to answer without referring back to the document on screen. These included nine multiple choice questions about each document (questions on the title, main idea and structure, three main factual questions and three incidental questions) followed by 10 recognition questions. There were no time limits on answering questions or any other part of the experiment.

\section{Results}

A method for selecting appropriate transforms proposed by Kirk (1995) was applied to comprehension scores and reading rates. This method follows general rules based on which transformation is most successful in a given situation. Angular transformations have been applied to proportions of perfect performance, where there is a maximum limit and the data are negatively skewed. An angular transformation is used to transform the scores to a normal distribution, suitable for parametric statistics. Answers to the multiple choice comprehension questions were therefore scored as a proportion of correct responses and then transformed $(2 \arcsin V)$ for statistical analysis. In contrast, when times are recorded, the measure has a minimum limit, but no maximum and the data can be positively skewed. Logarithmic transformations have been found to be useful in these circumstances. Reading rates in words per second were transformed by log (words per second +1$)$.

\subsection{COMPREHENSION}

The recognition scores were adjusted to make comparisons across the question types. This was necessary as chance level of performance for the multiple choice questions is 0.33 (as three alternatives were available), whereas chance is 0.5 for the recognition questions. The adjustment standardized chance level at 0.33 for all questions, so that scores became relative to their distance above or below chance. In calculating an overall comprehension score, each score was weighted according to the number of questions of that type. Following this adjustment and the angular transformation, chance is equal to approximately $1.57(\pi / 2)$ and a perfect score is approximately $3.14(\pi)$ for all question types and for overall comprehension.

\subsubsection{Reading speed and line length. A two-way analysis of variance on comprehension} scores, with reading speed as a between subject factor and line length as a within subject factor, found a main effect of reading speed on overall comprehension $(F(1,34)=22.98$, $p<0.0001)$ with a drop in comprehension at the faster speed. 


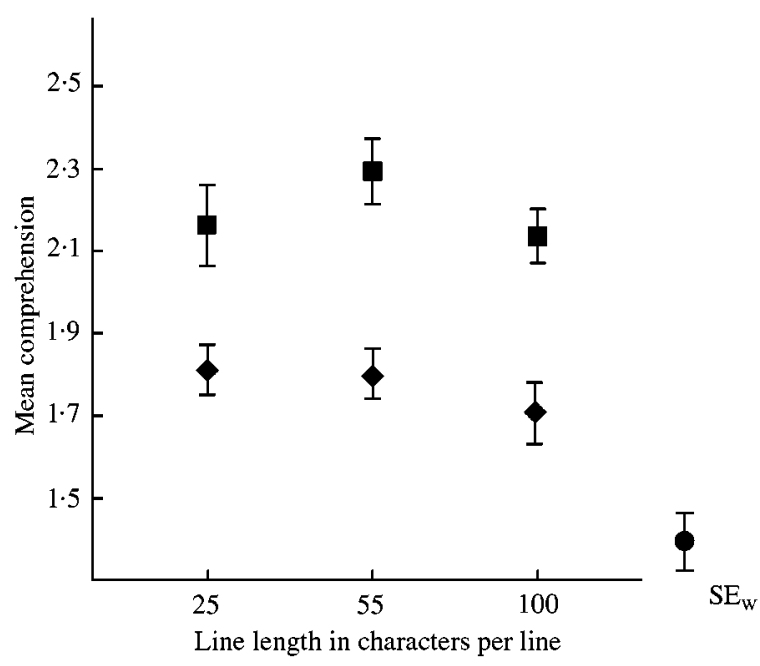

FIGURE 2. Mean comprehension scores across three line lengths when reading at normal and fast speed.

The second factor in the ANOVA was also significant producing a main effect of line length on overall comprehension $(F(2,68)=3.17, p<0.05)$. The data are illustrated in Figure 2. The standard error bars on each data point give an indication of the variability between subjects. $\mathrm{SE}_{\mathrm{w}}$ is the standard error of within subject comparisons. Post hoc multiple comparisons using Newman-Keuls test showed that comprehension of $55 \mathrm{cpl}$ documents was significantly better than $100 \mathrm{cpl}$ documents $(p<0.05)$. No other differences between line lengths were statistically significant. There was no interaction between reading speed and line length.

4.1.2. Reading rate. A series of correlations was carried out to assess whether there was any relationship between the time an individual spent in reading a document within each reading speed and their comprehension (between individual correlation). At the fast reading speed, with all line lengths combined, there was only one significant negative correlation between reading rate and incidental questions (Pearson correlation coefficient, $R=-0.23, p<0.02$ ). At the normal reading speed, there was a positive correlation between reading rate and overall comprehension $(R=0.21, p<0.03)$.

4.1.3. Nature of comprehension. A two-way ANOVA with reading speed (between subject) and question type (within subject) as factors found differences in comprehension depending on question types $(F(5,170)=7.12, p<0.0001)$. Comprehension scores for each question type are shown in Figure 3. The standard error bars for title, main idea and structure questions are larger as the data points are based on fewer questions. The data are averaged across the two reading speeds as there is no interaction between speed and question type. Using Newman-Keuls, structure questions were found to be significantly more difficult than all other question types $(p<0.05)$ and main factual questions were answered better than recognition questions $(p<0.05)$. 


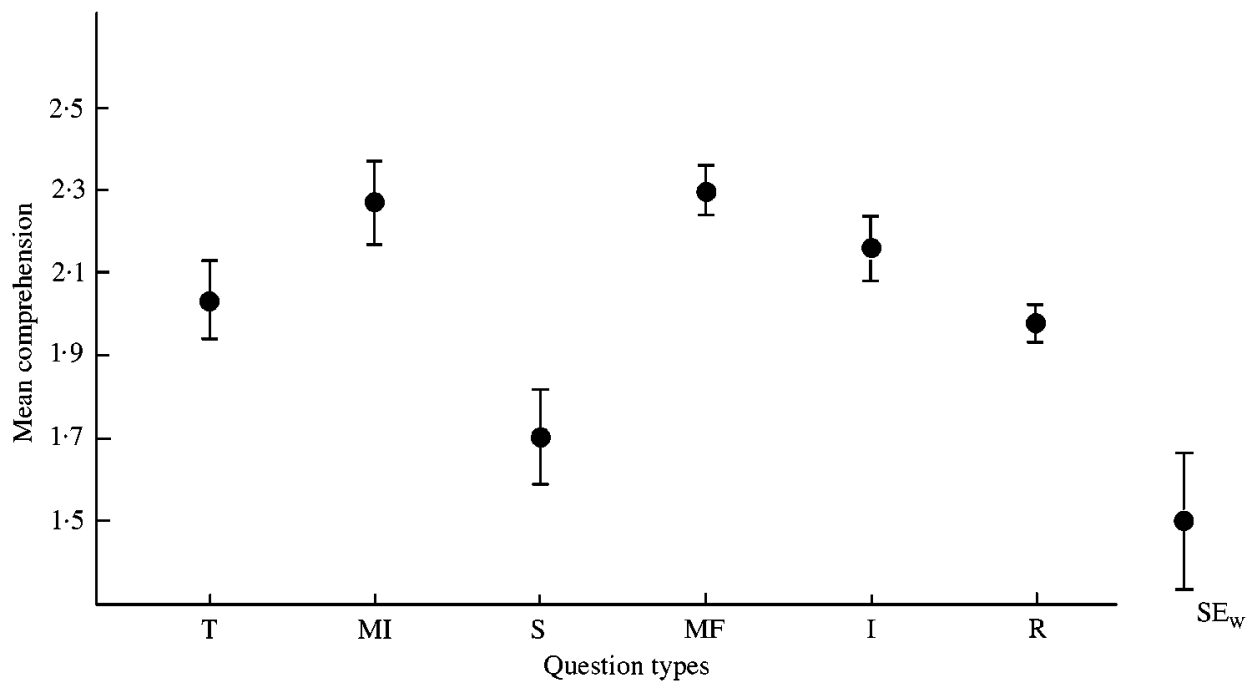

FIGURE 3. Mean comprehension scores according to question type. T title; MI Main idea; S structure; MF main factual; I incidental; $\mathrm{R}$ recognition.

\subsection{READING RATE}

The mean reading rate in words per second for the fast condition was 6.29 and 2.52 for the normal reading condition (377 and 151 words/min).

4.2.1. Reading speed and line length. A two-way ANOVA on reading rate including reading speed and line length as factors found the expected main effect of reading speed $(F(1,34)=56.06, p<0.0001)$, confirming that participants in the fast reading condition were reading faster, and there is a clear separation between the two speeds. There was also a main effect of line length $(F(2,68)=19.63, p<0.0001)$ and an interaction between reading speed and line length $(F(2,68)=7.18, p<0.002)$. The data are illustrated in Figure 4. At the fast reading speed, documents of 55 and $100 \mathrm{cpl}$ are read significantly faster than documents of $25 \mathrm{cpl}$. At the normal reading speed, $55 \mathrm{cpl}$ documents are read faster than $25 \mathrm{cpl}$ documents. All differences are significant at $p<0.05$ using Newman-Keuls.

\subsection{COMBINED COMPREHENSION AND READING RATE}

A multivariate analysis of variance was used to consider the effects of speed and line length on both reading rate and comprehension. This test takes into account possible correlations between the two measures. Winer (1971) suggests that it can be considered as a univariate test on optimally weighted composites of the individual variables. This analysis was chosen in preference to the measure of effective reading used by Jackson and McClelland (1979) which multiplies speed and comprehension. As these authors point out, multiplication may exaggerate the reading ability of very fast readers. The experimental manipulation in this study necessarily produced fast reading. 


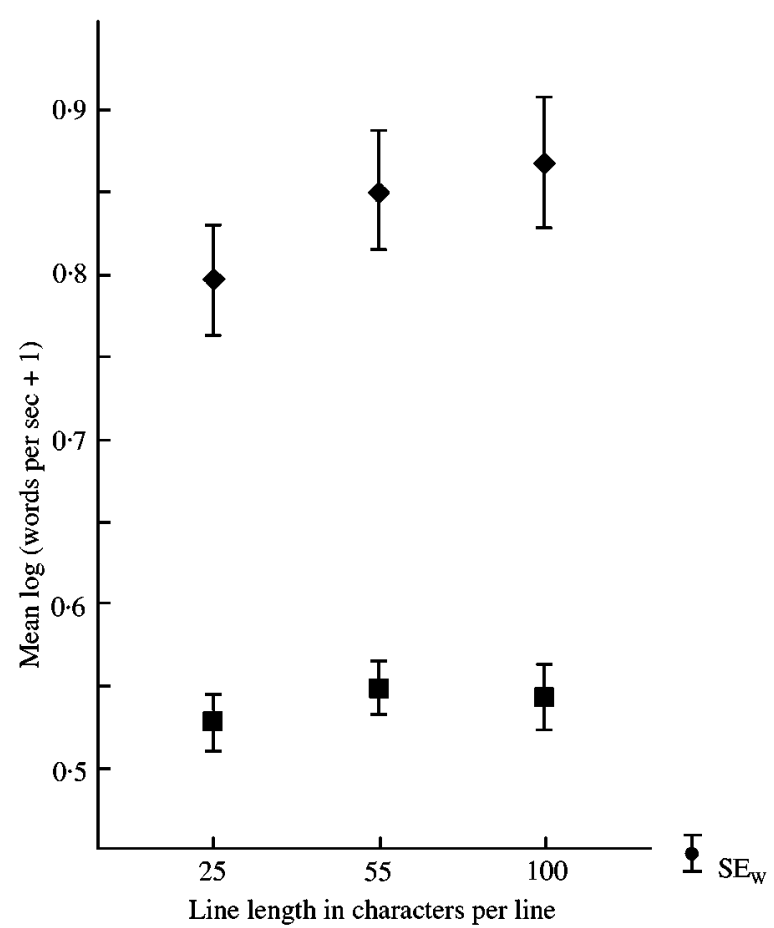

FIGURE 4. Mean reading rates across three line lengths when reading at normal and fast speed.

4.3.1. Reading speed and line length. The two-way MANOVA included reading speed as a between subject factor and line length as a within subject factor (as in the earlier ANOVAs). This analysis found a main effect of speed, using Wilk's Lambda, $(F(2,33)=39.06, p<0.0001)$. There was also a main effect of line length $(F(4,31)=9.54$, $p<0.0001)$ and a weaker interaction between speed and line length $(F(4,31)=3.33$, $p<0.03)$. At each line length, there are significant differences between performance at the two speeds $(F(6,29)=12.27, p<0.0001)$ in terms of both reading rate and comprehension. Similarly, at each speed, there are significant differences between line lengths (Fast: $F(4,14)=5.83, p<0.006$; Normal: $F(4,14)=6.11, p<0.005)$. These results are in line with the univariate analyses, which can be expected when correlations between dependent variables are relatively weak.

\subsection{SCROLLING PATTERNS}

The record of keystrokes used to move through the documents was summarized in a number of ways. When participants scrolled through the document, the time of each key press was recorded and these were grouped into discrete scrolling movements with pauses in between. A movement was defined as single or multiple key presses that were separated from other key presses by at least $3 \mathrm{~s}$. Any key presses that followed on from other presses within $2 \mathrm{~s}$ were regarded as part of the single movement (cf. de Bruijn et al., 1992). 
Scrolling up or back tracking was not very common. In the fast condition, one participant in particular accounted for most of the upward scrolling movements, which were very few. Reading at a normal speed, there were more upward movements spread across different participants, but there was no obvious pattern across line lengths. Therefore, in looking at scrolling patterns, both up and down movements have been grouped together as both contribute to the time spent scrolling.

For each participant, the times spent in pauses and scrolling movements, the number of scrolling movements, and the length of the first pause were recorded for each line length. The first pause was included to see whether the time spent before scrolling might be a characteristic of certain scrolling patterns. As the numbers of lines in documents vary according to line length, some calculations took the number of lines into account. This ensured that any conclusions based on comparisons of scrolling patterns across line lengths would not be confounded by the different amount of scrolling required.

In relation to scrolling movements, the speed of scrolling was calculated by dividing the number of lines in the document by the time spent in scrolling movements, resulting in a number of lines per second. The size of scrolling movements or scrolling length, was based on the number of lines in the document divided by the number of movements. The frequency of scrolling comes from the number of movements divided by the time in movement. The transformation applied to the above measures was selected by using a second approach described by Kirk (1995). As the nature of the distribution of values for these variables was unclear, it was not possible to follow general rules, as described at the beginning of the results section. Instead, the procedure identifies the transformation which produces the smallest ratio between the largest and smallest range of scores across treatments. This identified the log transformation as the most appropriate to apply to all three measures: speed of scrolling, size of scrolling movements and frequency of scrolling.

The time spent in pausing was not adjusted in any way as the number of words in each document remains constant across line length and none of the transformations proposed by Kirk (1995) decreased the range of scores. The pause to scrolling ratio describes the times in pauses divided by the time in movement per line. This variable therefore takes account of the different amount of scrolling required at the different line lengths. Again, no transformation was applied as these did not reduce the range of scores.

4.4.1. Reading speed and line length. Means and standard deviations for each reading speed are provided in Tables 2 and 3, broken down into line lengths. Two-way analyses of variance were computed for each of the five scrolling pattern variables summarized in the tables. Speed was included as a between subject factor and line length as within subject. Significant main effects $(p<0.05)$ are indicated by symbols in the two tables. $\mathrm{A} \dagger$ against the scrolling pattern variable in each table heading indicates a main effect of speed on this variable. A $\$$ within the table indicate that the line length is significantly different to the other two line lengths.

4.4.1.1. Scrolling speed, scrolling length and scrolling frequency. There were no significant effects in relation to speed of scrolling. However, there was a main effect of speed on scrolling length $(F(1,34)=4.25, p<0.05)$, with participants using shorter scrolling 
TABLE 2

Means and standard deviations of scrolling pattern variables at fast speed

\begin{tabular}{|c|c|c|c|c|c|c|c|c|c|c|}
\hline \multirow[b]{2}{*}{ LL (cpl) } & \multicolumn{2}{|c|}{ SS } & \multicolumn{2}{|c|}{$\mathrm{SL} \dagger$} & \multicolumn{2}{|c|}{$\mathrm{SF}$} & \multicolumn{2}{|c|}{$\mathrm{PT} \dagger$} & \multicolumn{2}{|c|}{$\mathrm{PS} \dagger$} \\
\hline & Mean & S.D. & Mean & S.D. & Mean & S.D. & Mean & S.D. & Mean & S.D. \\
\hline 25 & 0.63 & 0.16 & 1.27 & 0.24 & 0.08 & 0.04 & 133.39 & 97.48 & 519.18 & 441.95 \\
\hline 55 & 0.62 & 0.15 & 1.05 & 0.27 & 0.13 & 0.08 & 144.47 & 87.68 & 505.91 & 375.36 \\
\hline 100 & 0.66 & 0.13 & 1.04 & 0.27 & 0.14 & 0.07 & $157.14 t$ & 89.68 & 581.84 & 372.39 \\
\hline
\end{tabular}

$\dagger$ Indicates significant main effect of speed.

\$Indicates that line length is significantly different to the other two line lengths.

Note: LL, line length; SS, scrolling speed; SL, scrolling length; SF, scrolling frequency; PT, pause time; and $\mathrm{PS}$, pause to scrolling ratio.

TABLE 3

Means and standard deviations of scrolling pattern variables at normal speed

\begin{tabular}{|c|c|c|c|c|c|c|c|c|c|c|}
\hline \multirow[b]{2}{*}{ LL (cpl) } & \multicolumn{2}{|c|}{ SS } & \multicolumn{2}{|c|}{$\mathrm{SL} \dagger$} & \multicolumn{2}{|c|}{ SF } & \multicolumn{2}{|c|}{$\mathrm{PT} \dagger$} & \multicolumn{2}{|c|}{$\mathrm{PS} \dagger$} \\
\hline & Mean & S.D. & Mean & S.D. & Mean & S.D. & Mean & S.D. & Mean & S.D. \\
\hline 25 & 0.69 & 0.09 & 1.21 & 0.24 & 0.11 & 0.04 & 370.31 & 96.13 & 1471.25 & 469.23 \\
\hline 55 & 0.70 & 0.10 & 1.26 & 0.30 & 0.10 & 0.07 & 371.78 & 93.47 & 1542.82 & 532.22 \\
\hline 100 & 0.70 & 0.11 & 1.33 & 0.34 & 0.09 & 0.06 & $390.81 \%$ & 104.33 & 1597.00 & 613.52 \\
\hline
\end{tabular}

$\dagger$ Indicates significant main effect of speed.

$\$$ Indicates that line length is significantly different to the other two line lengths.

Note: LL, line length; SS, scrolling speed; SL, scrolling length; SF, scrolling frequency; PT, pause time; and $\mathrm{PS}$, pause to scrolling ratio.

movements when reading at the fast speed. There was also an interaction between speed and line length on scrolling length $(F(2,68)=9.35, p<0.001)$. Scrolling lengths are similar in all line lengths at the normal reading speed. However when reading fast, longer scrolling movements are used at $25 \mathrm{cpl}$. At this line length there is little difference in the size of the scrolling movements at the two speeds. Whereas at 55 and $100 \mathrm{cpl}$ participants use longer scrolling movements when reading at a normal speed, compared with a fast speed. There is also an interaction between speed and line length $(F(2,68)=5.02$, $p=0.01$ ) on scrolling frequency. As with scrolling length, frequency of scrolling is similar across line lengths at the normal speed, but when reading fast, scrolling is less frequent at the short line length.

4.4.1.2. Pause time and pause to scrolling ratio. There is a main effect of speed on the time spent in pauses $(F(1,34)=59.38, p<0.001)$. Participants spend less time pausing when required to read fast. There is also a main effect of line length $(F(2,68)=5.15$, $p=0.01)$. A follow-up test with Newman Keuls identifies a significant difference between $100 \mathrm{cpl}$ and the two shorter line lengths, 25 and $55 \mathrm{cpl},(p<0.05)$, but no difference between 25 and $55 \mathrm{cpl}$. Pauses are longer at the longer line length. When comparing the 
time spent pausing in relation to scrolling, there is a main effect of speed $(F(1,34)=52.95$, $p<0.001)$. At the fast speed, relatively less time is spent pausing compared with scrolling. There are no significant differences between line lengths, having taken into account the amount of scrolling required (number of lines).

4.4.2. Reading rate and scrolling patterns. Multiple regressions were used to explore whether differences in reading rate are associated with patterns of scrolling and participants' age and reported reading behaviour. The measures used in relation to scrolling patterns were time in pauses and movements, number of movements and length of the first pause. It was unnecessary to use the measures above, which take into account the number of lines in each document, as the statistics were calculated separately for each line length. Fast and normal reading speeds were also treated separately.

The regression identifies the combination of variables which produces the highest adjusted $R^{2}$ (adjusted for the number of variables in the model). Table 4 lists the variables which account for differences in reading rate between individuals at the fast reading speed, and Table 5 the normal reading speed. The variables are listed in decreasing order of statistical significance, and individual variables making a significant contribution $(p<0.05)$ are marked with $\mathrm{a} \uparrow$. The numbers in parentheses show the relative importance of the variable effects when not affected by scales of measurement (standardized regression coefficients).

4.4.3. Comprehension and scrolling patterns. Any relationships between scrolling patterns, participants' characteristics and resulting comprehension scores were also investigated with multiple regressions. Reading rate is included as an additional variable to estimate its importance in relation to the other variables. Table 6 lists the variables contributing to differences in overall comprehension between individuals at the fast reading speed, and Table 7 lists the equivalent data for the normal reading speed.

\section{Discussion}

\subsection{COMPREHENSION}

Comprehension is better when reading at a normal speed, indicating a speed-accuracy trade-off, as found by Dyson and Haselgrove (2000). Our increase in reading speed is

TABLE 4

Variables which account for differences in reading rate at fast reading speed

\begin{tabular}{|c|c|c|}
\hline LL (cpl) & $R^{2}$ & Variables in model \\
\hline 25 & 0.94 & $\mathrm{TP} \dagger(-0.98), \mathrm{TM} \dagger(-0.47), \operatorname{AGE} \dagger(0.11), \mathrm{NM}(-0.08)$ \\
\hline 55 & 0.94 & $\mathrm{TP}+(-0.96), \mathrm{TM}+(-0.3), \mathrm{AGE}+(0.09)$ \\
\hline 100 & 0.97 & 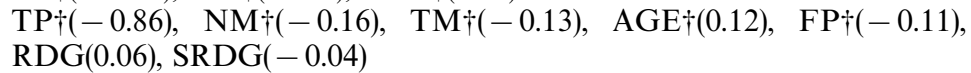 \\
\hline
\end{tabular}

Note: TP, time in pauses; TM, time in movements; NM, number of movements; FP, length of first pause; AGE, age band (18-24 or 25-44); RDG, reported frequency of reading; and SRDG, reported frequency of screen reading. 
TABLE 5

Variables which account for differences in reading rate at normal reading speed

\begin{tabular}{ccl}
\hline LL $(\mathrm{cpl})$ & $R^{2}$ & \multicolumn{1}{c}{ Variables in model } \\
\hline 25 & 0.98 & $\mathrm{TP} \dagger(-1.09), \mathrm{TM} \dagger(-0.23), \mathrm{FP} \dagger(0.12), \mathrm{NM} \dagger(0.17)$ \\
55 & 0.98 & $\mathrm{TP} \dagger(-1), \mathrm{TM} \dagger(-0.07), \mathrm{FP}(0.05), \mathrm{RDG}(0.04), \mathrm{NM}(0.04)$, \\
100 & 0.97 & $\mathrm{TP} \dagger(-1), \mathrm{TM} \dagger(-0.1), \mathrm{FP}(0.05)$ \\
\hline
\end{tabular}

Note: TP, time in pauses; TM, time in movements; NM, number of movements; FP, length of first pause; RDG, reported frequency of reading.

TABLE 6

Variables which account for differences in overall comprehension at fast reading speed

\begin{tabular}{ccl}
\hline LL $(\mathrm{cpl})$ & $R^{2}$ & \multicolumn{1}{c}{ Variables in model } \\
\hline 25 & 0.21 & $\mathrm{TP} \dagger(1.24), \mathrm{SRDG} \dagger(0.47), \mathrm{FP} \dagger(-0.64), \mathrm{RDG}(0.3), \mathrm{RR}(0.5)$ \\
55 & 0.12 & $\mathrm{TM} \dagger(0.42), \mathrm{TP}(0.17)$ \\
100 & 0.47 & $\mathrm{NM} \dagger(0.6), \mathrm{TM}(0.21), \mathrm{FP}(0.17)$ \\
\hline
\end{tabular}

Note: $\mathrm{RR}$, reading rate; TP, time in pauses; TM, time in movements; NM, number of movements; FP, length of first pause; RDG, reported frequency of reading; and SRDG, reported frequency of screen reading.

TABLE 7

Variables which account for differences in overall comprehension at normal reading speed

\begin{tabular}{cll}
\hline LL (cpl) & $R^{2}$ & \multicolumn{1}{c}{ Variables in model } \\
\hline 25 & 0.17 & $\mathrm{RR}(1.41), \mathrm{FP}(0.31), \mathrm{AGE}(0.25), \mathrm{TP}(1.14)$ \\
55 & 0.1 & $\mathrm{SRDG} \dagger(0.36), \mathrm{FP}(0.18)$ \\
100 & 0.32 & $\mathrm{RR} \dagger(0.72), \mathrm{FP} \dagger(0.64), \mathrm{RDG} \dagger(-0.4), \operatorname{AGE}(0.27), \mathrm{NM}(0.27)$ \\
\hline
\end{tabular}

Note: RR, reading rate; TP, time in pauses; NM, number of movements; FP, length of first pause; AGE, age band (18-24 or 25-44); RDG, reported frequency of reading; and SRDG, reported frequency of screen reading.

likely to be greater than the 'mild time pressure' that has been shown to improve comprehension (Walczyk et al., 1999). Although the current study used a between group design, the results appear to parallel the finding that comprehension decreases when an individual increases his or her reading rate (Poulton, 1958).

In contrast, faster readers within the normal reading speed group appear to recall more at the normal reading speed. This between individual correlation has also been reported elsewhere (e.g. Jackson \& McClelland, 1979; Carver, 1990). However, correlations between individual reading rates and comprehension are rather weak. There is an indication of a slight speed-accuracy trade-off at the fast reading speed, particularly in relation to the recall of details (cf. Just \& Carpenter, 1987). However, given the extent of reading acceleration required for the fast condition, it is plausible that variations in individual reading rates have relatively little additional effect on comprehension.

In this study, line length influences readers' comprehension with documents at $55 \mathrm{cpl}$, the medium line length, producing better comprehension scores than the longest line length $(100 \mathrm{cpl})$. This result was not found in earlier studies of the effect of line length on 
comprehension (Duchnicky \& Kolers, 1983; Dyson \& Kipping, 1998), but the difference may be accounted for by the more elaborate test of comprehension. Requiring participants to recall details and make inferences is likely to have produced a more sensitive measure of comprehension that is able to detect differences in levels of comprehension.

The explanation for better comprehension at $55 \mathrm{cpl}$ than $100 \mathrm{cpl}$ may be tied in with the nature of eye movements and the potential problem of locating the beginning of a line, following a return sweep at long line lengths. Although we would expect this factor to have a more direct effect on reading rate, interruptions to reading may conceivably disrupt concentration and hence impair comprehension. The reported inefficiency of eye movements at very short lines does not translate into lower comprehension, but may affect reading rate.

Differences between question types were similar to the earlier study (Dyson \& Haselgrove, 2000). The questions relating to the structure or order of items within the texts were the most difficult questions to answer. Readers were unable to locate specific content (e.g. ideas, events) in relation to other information. It is possible that the scrolling display contributed to the difficulty in answering these questions. Cues to location that are available in print, or in paged screen displays (e.g. near the top of the page) are lost when text is scrolled within a window (see Piolat et al., 1997).

Main factual questions that relate to the more important units of the text were answered more easily than those requiring the recall of specific details (recognition questions). This difference was demonstrated by Wagner and Sternberg (1987), who found that questions on the gist and main ideas were answered more accurately than questions on detail and those requiring analysis. Although answered reasonably well, questions requiring inferences were not significantly better than other types in this study, as they were in Dyson and Haselgrove (2000).

Performance across different question types and reading speeds did confirm the finding of Dyson and Haselgrove (2000) that the type of information recalled is not dependent on reading speed. Significantly increasing reading speed affected all types of information. However, at the fast reading speed, faster readers were poorer at recalling incidental material, which was also found in the McConkie et al. (1973) study. Participants' familiarity with the range of questions they were required to answer may have contributed to this consistency. Knowing what types of questions they would be asked, they may have tried to maximize their chances of being able to answer all of them, whatever the reading speed. Practice trials introduced the nature of questions used in the experiment and subsequent trials during the course of the experiment would have reinforced this pattern of questioning.

\subsection{READING RATE}

The reading rates measured in this study are slower than the equivalent rates in Dyson and Haselgrove (2000). However, the previous study differed by using a within subject design and only one line length $(60 \mathrm{cpl})$, which would have introduced less uncertainty. McConkie et al. (1973) found that uncertainty in relation to question type slowed down reading. Line length may have a similar effect.

This experiment replicates the finding of Dyson and Kipping (1998) that documents presented in a narrow column $(25 \mathrm{cpl})$ are read slower than longer line lengths. However, 
unlike the earlier study, increasing line length from 55 to $100 \mathrm{cpl}$ does not improve reading rate. The effect of line length on reading rate is also dependent on the overall reading speed. Particularly long lines $(100 \mathrm{cpl})$ are only read faster than the short lines when already reading at a fast speed. Across both speeds, $55 \mathrm{cpl}$ documents are read the fastest.

With a short line length, more time is necessarily spent in scrolling as the document is longer. Also, readers may not be able to make use of much information in each fixation and may decrease their saccade length (Rayner \& Pollatsek, 1989). This would slow down reading. The advantage of long lines only at the fast speed may indicate that this format facilitates skim reading, along with $55 \mathrm{cpl}$, but is not an advantage when reading at a normal speed. This may simply be explained by the mechanics of scrolling, i.e. less action required, which are accentuated at a fast speed. Alternatively, the advantage of long lines may be in displaying more information at a time. If readers are not concerned with reading every word, this format allows for easier skimming. However, this can be at the expense of comprehension.

\subsection{COMBINED COMPREHENSION AND READING RATE}

The multivariate analysis confirms that the differences in comprehension at varying line lengths are not cancelled out by differences in reading rate (i.e. speed-accuracy trade-off). When the two measures are combined, line length is still a significant factor.

\subsection{SCROLLING PATTERNS}

The consistency in scrolling speed across reading speeds is interesting, as a possible means of increasing reading speed would be to scroll faster. At fast reading speeds participants are generally making smaller scrolling movements which Dyson and Kipping (1998) found corresponded with increased time in movement. As participants were not under time pressure in the earlier study, this could account for the difference. In the current study, the urgency imposed by the requirement to read faster than normal may have prompted the smaller movements. These results suggest that either participants cannot increase their scrolling speeds or do not know how to do so, or they do not consider this an effective strategy for reading faster. If participants read whilst scrolling, increasing the scrolling speed could be counter productive. The means by which participants increase their reading speed appears to be by reducing the time they spend pausing between movements. This is reinforced by the reduction in the ratio of pausing to scrolling at fast speeds.

Line length influences the time spent in pauses, with the longest pauses at the longest line length. Participants may feel they can allocate more time as they need not spend as much time in scrolling. Alternatively, this format may require more time to read.

The effect of reading speed on the size and frequency of scrolling movements is dependent upon line length. Although overall participants are making smaller movements when reading fast, with short line lengths at this speed they are making slightly larger and less frequent scrolling movements, similar to the patterns found across all line lengths at normal reading speed. This similarity does not appear to be due to a ceiling effect whereby a maximum length is reached as faster reading did not generally produce 
longer scrolling movements. Instead, it is likely that characteristics of the short line length are relevant. This line length requires more scrolling to get to the bottom of the document. Small movements will reveal relatively little new text and would therefore be an inefficient strategy for increasing speed of reading. Participants' reading rates at both speeds are slower at this line length. As mentioned above, this may be partly attributable to the increase in time required for scrolling.

5.4.1. Reading rate and scrolling patterns. The time spent in pausing between scrolling movements is clearly the main determinant of reading rate. Those participants who read faster spent less time in pauses. The time spent in scrolling movements is generally the next most important variable, with those spending less time scrolling reading faster. Although the age of participants appears to be relevant when reading fast, this result is based on a limited range of ages and a small sample.

5.4.2. Comprehension and scrolling patterns. Identifying the variables that contribute to individuals' comprehension scores is much more difficult than identifying reading rate predictors. The adjusted $R^{2}$ values from multiple regressions are much lower than those for reading rate, so less of the variability in comprehension is accounted for by these variables. $R^{2}$ values are higher at the fast reading speed suggesting that the pattern of reading and participants' reported reading behaviour may have greater relevance when under time pressure. These values are also larger for documents at $100 \mathrm{cpl}$, again indicating that the way in which the document is read has a greater effect at this extreme.

At the fast reading speed, the variables accounting for differences in comprehension between individuals vary across line lengths. However, as $R^{2}$ values also differ the more significant findings are selected for discussion. When reading the narrow column, participants who spent longer in pauses, although with a shorter initial pause, had better comprehension scores. De Bruijn et al. (1992) suggested that attending to local information for longer could help with integrating information on a small screen. Overall time spent pausing also produced better comprehension scores in the previous study by Dyson and Haselgrove (2000). However, this approach may be at the expense of reading rate, as those who spent longer in pauses have a slower reading rate. With long line lengths, the method of scrolling is particularly relevant. Higher comprehension is found amongst people who read with a greater number of individual scrolling movements. Again, this scrolling pattern is inefficient in terms of increasing reading speed.

If we assume that readers are not reading whilst scrolling, the benefit of longer pauses at short line lengths comes from longer time to read, i.e. individuals are trading speed for accuracy. At long line lengths, the size of the chunk of text available for reading is potentially much larger. Hence, breaking up the reading process by more frequent scrolling movements, without reading, may allow time for consolidation (cf. Poulton, 1958). Also by scrolling more frequently the extent of individual movements can be reduced, which may make it easier for readers to re-locate their position in the text. This may be particularly helpful with long line lengths as less vertical movement may ease the difficulty of locating the appropriate line when reading quickly. There may therefore be less distraction to the process of reading from the mechanics of the movements (Hansen \& Haas, 1988; O’Hara \& Sellen, 1997). This latter explanation would also apply if readers were continuing to read whilst scrolling, as moving the document by smaller amounts 
should make it easier to maintain their position in the text. At shorter line lengths, this pattern of reading may not be such an effective strategy. It would result in much more time spent reading whilst the text is scrolled, whereas the time in pauses is a better predictor of comprehension at short line lengths.

When reading at a normal speed, only the longest line length provides reasonably consistent data. At $100 \mathrm{cpl}$ the importance of reading rate as a predictor of comprehension confirms the finding that faster readers recall more information. Although a longer initial pause is also correlated with better comprehension, other aspects of the scrolling pattern, i.e. scrolling movements, seem less relevant. This contrasts with reading $100 \mathrm{cpl}$ documents at a fast rate where the method of scrolling is important. The speed appears to be accentuating the importance of how text is scrolled. How the document is navigated therefore appears to be critical when it is more difficult to recall information, i.e. when reading at a fast speed and when documents have long lines.

\section{General discussion and conclusions}

By reading almost twice as fast as normal we increase the volume of material we get through on screen but acquire a less complete account of a document. Faster readers reading at their normal speed, can recall more than slower readers, but this does not mean that encouraging someone to read faster will improve their comprehension.

In circumstances where readers know they will be asked a range of question types, faster reading does not adversely affect recall of particular types of information. However, at both normal and fast speeds, more general information is recalled better than specific detail. In particular, the order or position of items within the document is very poorly recalled.

The effects of line length on both comprehension and reading rate may be explained in terms of the mechanics of reading (eye movements), mechanics of scrolling and consolidation of what is read. Both very short and very long line lengths can slow down reading through disrupting the normal pattern of eye movements. Reading short line lengths places greater demands on scrolling time, but with long lines the time spent in scrolling may enable consolidation of what has been read.

A line length of $55 \mathrm{cpl}$ appears to support effective reading in terms of both rate and comprehension. However, as the line lengths used in this study were spread across a wide range, there may be a more optimal setting than this. By varying the range and extremes of line lengths in future research, it may be possible to more precisely identify an optimal format and to explore the relative contributions of mechanical and cognitive factors.

When reading under more difficult circumstances (e.g. time pressure or extreme line lengths), the way in which the document is read appears to have more influence on comprehension than when reading normally. Readers increase their reading rate by reducing time between scrolling and also modifying the nature of their scrolling movements. The effectiveness of this strategy in maximizing comprehension may be dependent upon the extent to which people read during scrolling. This issue emerged within the discussion of scrolling patterns and is further developed below. However, as this study did not specifically set out to identify when reading takes place in relation to navigation, no conclusions are drawn. 
One indication that participants may read during scrolling is the trade-off between pause time and scrolling time (longer pauses at $100 \mathrm{cpl}$ ). If people are not reading whilst scrolling, they are spending a lot less time reading the shorter line lengths. If this were the case, we might expect poorer comprehension at 25 and $55 \mathrm{cpl}$, which was not found.

The consolidation explanation assumes that readers are not reading during scrolling. This explanation was introduced to explain the advantage of frequent scrolling movements at long line lengths. There is some evidence that reading may take place whilst scrolling at shorter line lengths, and not with longer line lengths (Dyson \& Kipping, 1998).

Perhaps not surprisingly, there is no simple description of an effective reader. There appears to be a difference between methods of scrolling and pausing that are exhibited by faster readers and those who have good comprehension scores. Fast readers, at both normal and fast speeds, spend less time pausing between fewer scrolling movements. However at fast speeds, better comprehension is found amongst readers who spend longer in pauses $(25 \mathrm{cpl})$ or use more scrolling movements $(100 \mathrm{cpl})$. A definition of effective reading therefore needs to differentiate between faster reading and more accurate recall of what is read. The nature of individual reading tasks may determine which is more important.

Further research can usefully explore the effects of manipulating scrolling patterns to determine whether changing individuals' patterns of pausing and scrolling can affect comprehension. The current results suggest that such experiments should also include factors that may increase reading difficulty.

With these data we should be in a better position to inform the design of future interfaces for reading from screen. Digital genres are just starting to emerge (Dillon \& Gushrowski, 2000), but they may not have fixed formats unlike printed documents (e.g. newspapers). Indeed, web browsers, by enabling us to manipulate the size of windows, discourage the emergence of conventional forms which might include line length. This flexibility afforded by current browsers also makes it difficult to control presentation so that readers might benefit from the results of this research. Currently, this control is available through style sheets, so that informed web page designers/authors will be able to define formats that may be more effective for certain types of reading. However, a question remains as to whether we should constrain our readers, which may return us to the inflexibility of print, or continue to exploit the flexibility inherent in the medium.

This research was supported by a grant from Microsoft Corporation.

\section{References}

Belmore, S. M. (1985). Reading computer-presented text. Bulletin of the Psychonomic Society, 23, $12-14$.

BREZNITZ, Z. (1997). Effects of accelerated reading rate on memory for text among dyslexic readers. Journal of Educational Psychology, 89, 289-297.

Brown, A. L. \& SMILEY, A. S. (1977). Rating the importance of structural units of prose passages: a problem of metacognitive development. Child Development, 48, 1-8.

CARVER, R. P. (1990). Reading Rate: a Review of Research and Theory. San Diego: Academic Press.

De Bruijn, D., De Mul, S. \& Van Oostendorp, H. (1992). The influence of screen size and text layout on the study of text. Behaviour \& Information Technology, 11, 71-78. 
Dillon, A. (1992). Reading from paper versus screens: a critical review of the empirical literature. Ergonomics, 35, 1297-1326.

Dillon, A. \& Gushrowski, B. (2000) Genres and the Web-is the home page the first digital genre? Journal of the American Society for Information Science, 51, 202-205.

DUCHNICKY, R. L. \& KOLERS, P. A. (1983). Readability of text scrolled on visual display terminals as a function of window size. Human Factors, 25, 683-692.

Dyson, M. C. \& Haselgrove, M. (2000). The effects of reading speed and reading patterns on our understanding of text read from screen. Journal of Research in Reading, 23, 210-223.

Dyson, M. C. \& Kipping, G. J. (1997). The legibility of screen formats: are three columns better then one? Computers \& Graphics, 21, 703-712.

Dyson, M. C. \& Kipping, G. J. (1998). The effects of line length and method of movement on patterns of reading from screen. Visible Language, 32, 150-181.

Gould, J. D. \& GRISCHKOWSKY, N. (1984) Doing the same work with hard copy and cathode ray tube (CRT) computer terminals. Human Factors, 26, 323-337.

Gould, J. D., Alfaro, L., Barnes, V., Finn, R., Grischkowsky, N. \& Minuto, A. (1987). Reading is slower from CRT displays than from paper: attempts to isolate a single-variable explanation. Human Factors, 29, 269-299.

HANSEN, W. J. \& HAAS, C. (1988). Reading and writing with computers: a framework for explaining differences in performance. Communications of the ACM, 31, 1080-1089.

Harri-Augstein, S., Smith, M. \& Thomas, L. (1982). Reading to Learn. London: Methuen.

horton, W., TAYlor, L., Ignacio, A. \& Hoft, N. L. (1996). The Web Page Design Cookbook. New York: John Wiley.

JACKSON, M. D. \& MCCLELland, J. L. (1979). Processing determinants of reading speed. Journal of Experimental Psychology: General, 108, 151-181.

Johnson, R. E. (1970). Recall of prose as a function of the structural importance of the linguistic units. Journal of Verbal Learning and Verbal Behaviour, 9, 12-20.

Just, M. A. \& CARPENTER, P. A. (1987) The Psychology of Reading and Language Comprehension. Newton, MA: Allyn and Bacon.

KirK, R. E. (1995). Experimental Design: Procedures for the Behavioural Sciences. 3rd edn. Pacific Grove, USA: Brooks/Cole.

Masson, M. E. J. (1982). Cognitive processes in skimming stories. Journal of Experimental Psychology: Learning, Memory and Cognition, 8, 400-417.

Masson, M. E. J. (1985). Rapid reading processes and skills. In G. E. MackinNON \& T. G. Waller, Eds. Advances in Theory and Practice, Vol. 4, pp. 183-230. Orlando: Academic Press.

McConkie, G. W., Rayner, K. \& Wilson, S. J. (1973). Experimental manipulation of reading strategies. Journal of Educational Psychology, 65, 1-8.

MUter, P. (1996). Interface design and optimization of reading of continuous text. In H. VAN OostendorP \& S. De Mul, Eds. Cognitive Aspects of Electronic Text Processing, pp. 161-180. Norwood, NJ: Ablex.

Muter, P., Latremouille, S. A., Treurniet, W. C. \& Beam, P. (1982). Extended reading of continuous text on television screens. Human Factors, 24, 501-508.

Muter, P. \& MAurutTo, P. (1991). Reading and skimming from computer screens and books: the paperless office revisited? Behaviour \& Information Technology, 10, 257-266.

O'HARA, K. \& SEllen, A. (1997). A comparison of reading paper and on-line documents. In S. Pemberton, Ed. CHI 97 Conference: Human Factors in Computing Systems, pp. 335-342. Reading, MA: Addison-Wesley, ACM Press.

Piolat, A., Roussey, J.-Y. \& Thunin, O. (1997). Effects of screen presentation on text reading and revising. International Journal of Human-Computer Studies, 47, 565-589.

Poulton, E. C. (1958). Time for reading and memory. The British Journal of Psychology, 49, 230-245.

Rayner, K. \& Pollatsek, A. (1989). The Psychology of Reading. Hillsdale, NJ: Lawrence Erlbaum.

Robeck, M. C. \& Wallace, R. R. (1990). The Psychology of Reading: an Interdisciplinary Approach. Hillsdale, NJ: Lawrence Erlbaum. 
SMITH, A. \& SAVORY, M. (1989). Effects and after-effects of working at a VDU: investigation of the influence of personal variables. In E. D. MEGAW, Ed. Contemporary Ergonomics 1989, pp. 252-257. London: Taylor \& Francis.

SPENCER, H. (1968). The Visible Word. London: Royal College of Art.

Tinker, M. A. (1963). Legibility of Print. Ames: Iowa State University Press.

Wade, S. E., Schraw, G., Buxton, W. M. \& Hayes, M. T. (1993). Seduction of the strategic reader: effects of interest on strategies and recall. Reading Research Quarterly, 28, 93-111.

WAGNER, R. K. \& STERnberG, R. J. (1987). Executive control in reading comprehension. In B. K. Britton \& S. M. Glynn, Eds. Executive Control Processes in Reading, pp. 1-21. Hillsdale, NJ: Lawrence Erlbaum Associates.

WalczyK, J. J., Kelly, K. E., Meche, S. D. \& Brand, H. (1999). Time limitations enhance reading comprehension. Contemporary Educational Psychology, 24, 156-165.

WALLER, R. (1986) What electronic books have to be better than. Information Design Journal, $5,72-75$.

Winer, B. J. (1971). Statistical Principles in Experimental Design, 2nd edn. New York: McGraw-Hill.

ZhAI, S. \& SMITH, B. A. (1999). Multistream input: an experimental study of document scrolling methods. IBM Systems Journal, 38, 642-651.

Paper accepted for publication by Associate Editor, Ritu Agarwal

\section{Appendix: example of each type of question used to test comprehension}

Title

Which of the following titles best fits the text?
(a) Monaco's wealth and wealthy
(b) The racing and royalty of Monaco
(c) People and possessions in the principality of Monaco

Main idea

According to the author, what is Monaco dedicated to?

(a) The principle of fabulous excess

(b) Strength and the continuity of the monarchy

(c) Motor sport

\section{Structure}

Who does the author interview just before the description of Monaco's size (2.5 mile long strip), location and population?
(a) Prince Rainier III
(b) A British banker
(c) The Countess

\section{Incidental}

How long have the Grimaldis been in Monaco?

(a) Since 1940

(b) Since the 13th century

(c) Since the 19th century

Main factual

What ritual was described by the British banker?

(a) Showing off wealth to one another 
(b) Watching the Grand Prix

(c) Going to a nightclub

\section{Incidental}

What is the author taught to say in French?

(a) Corks for the ears

(b) I like jewels

(c) He's loaded

\section{Recognition}

Please indicate with a tick or cross whether or not each of the following appeared in the text you read:

$\square \quad$ One evening at dusk a full golden moon rose over the Mediterranean

$\square \quad$ The cars hurled past the belle époque buildings at the Place du Casinop

In Monaco no one does anything by half measures

Foreigners make up more than 80 percent of Monaco's official population of 30,000

A decent one bedroom apartment starts at $\$ 600,000$

To explain any natural hierarchy, it is necessary first to lay out the habitat

I sipped my drink, and faces began to materialize out of the darkness

But no one goes to Monaco to be unseen

They keep an apartment for tax purposes but actually live elsewhere

The box seats high up at the corners of the amphitheater belong to Monaco's elite 